\title{
re-Searcher: GUI-based bioinformatics tool for simplified genomics data mining of VCF files
}

\author{
Daniyar Karabayev ${ }^{\text {Equal first author, }{ }^{1} \text {, Askhat Molkenov }}{ }^{1}$, Kaiyrgali Yerulanuly ${ }^{1,2}$, Ilyas Kabimoldayev ${ }^{1}$, Asset Daniyarov ${ }^{1}$, \\ Aigul Sharip ${ }^{1}$, Ainur Seisenova ${ }^{1}$, Zhaxybay Zhumadilov ${ }^{1,3}$, Ulykbek Kairov ${ }^{\text {Corresp. Equal first author, } 1}$ \\ ${ }^{1}$ Laboratory of Bioinformatics and Systems Biology, Center for Life Sciences, National Laboratory Astana, Nazarbayev University, Nur-Sultan, Kazakhstan \\ 2 \\ 2 L.N. Gumilyov Eurasian National University, Nur-Sultan, Kazakhstan \\ 3 School of Medicine, Nazarbayev University, Nur-Sultan, Kazakhstan \\ Corresponding Author: Ulykbek Kairov \\ Email address: ulykbek.kairov@nu.edu.kz
}

Background. High-throughput sequencing platforms generate a massive amount of high-dimensional genomic datasets that are available for analysis. Modern and user-friendly bioinformatics tools for analysis and interpretation of genomics data becomes essential during the analysis of sequencing data. Different standard data types and file formats have been developed to store and analyze sequence and genomics data. Variant Call Format (VCF) is the most widespread genomics file type and standard format containing genomic information and variants of sequenced samples.

Results. Existing tools for processing VCF files don't usually have an intuitive graphical interface, but instead have just a command-line interface that may be challenging to use for the broader biomedical community interested in genomics data analysis. re-Searcher solves this problem by pre-processing VCF files by chunks to not load RAM of computer. The tool can be used as standalone user-friendly multiplatform GUI application as well as web application ( https://nla-lbsb.nu.edu.kz ). The software including source code as well as tested VCF files and additional information are publicly available on the GitHub repository (https://github.com/LabBandSB/re-Searcher). 
1 re-Searcher: GUI-based bioinformatics tool for 2 simplified genomics data mining of VCF files

3

4

5

6

7

\author{
Daniyar Karabayev 1,*, Askhat Molkenov ${ }^{1}$, Kaiyrgali Yerulanuly ${ }^{1,2}$, Ilyas Kabimoldayev ${ }^{1}$, Asset \\ Daniyarov $^{1}$, Aigul Sharip ${ }^{1}$, Ainur Seisenova ${ }^{1}$, Zhaxybay Zhumadilov ${ }^{1,3}$ and Ulykbek Kairov ${ }^{1, *}$ \\ ${ }^{1}$ Laboratory of Bioinformatics and Systems Biology, Center for Life Sciences, National \\ Laboratory Astana, Nazarbayev University, Nur-Sultan, Kazakhstan \\ ${ }^{2}$ L.N. Gumilyov Eurasian National University, Nur-Sultan, Kazakhstan \\ ${ }^{3}$ School of Medicine, Nazarbayev University, Nur-Sultan, Kazakhstan \\ *Equal first author \\ Corresponding Author: \\ Ulykbek Kairov $^{1}$ \\ 53 Kabanbay Batyr ave., Nur-Sultan, 010000, Kazakhstan \\ Email address: ulykbek.kairov@nu.edu.kz
}

\begin{abstract}
Background. High-throughput sequencing platforms generate a massive amount of highdimensional genomic datasets that are available for analysis. Modern and user-friendly bioinformatics tools for analysis and interpretation of genomics data becomes essential during the analysis of sequencing data. Different standard data types and file formats have been developed to store and analyze sequence and genomics data. Variant Call Format (VCF) is the most widespread genomics file type and standard format containing genomic information and variants of sequenced samples.
\end{abstract}

Results. Existing tools for processing VCF files don't usually have an intuitive graphical interface, but instead have just a command-line interface that may be challenging to use for the broader biomedical community interested in genomics data analysis. re-Searcher solves this problem by pre-processing VCF files by chunks to not load RAM of computer. The tool can be used as standalone user-friendly multiplatform GUI application as well as web application (https://nla-lbsb.nu.edu.kz). The software including source code as well as tested VCF files and additional information are publicly available on the GitHub repository (https://github.com/LabBandSB/re-Searcher).

\title{
Introduction
}

Recent achievements in high-throughput sequencing technologies (Goodwin, McPherson \& McCombie, 2016; van Dijk et al., 2018) have led to the generation of massive amounts of genomic data (Gao et al., 2019; The ICGC/TCGA Pan-Cancer Analysis of Whole Genomes Consortium, 2020) available for the research community. Many omics databases have been 
40 developed and have collected freely accessible datasets (Molkenov et al., 2019; Rigden \& 41 Fernández, 2020) for analysis by the bioinformatics community. Modern bioinformatics tools 42 and methods are in high demand for analyzing and interpreting the big omics data generated by 43 the different types of multi-omics platforms available. Different standard data types and file 44 formats have been developed to store and analyze sequence and genomics data. Variant Call 45 Format (VCF) (Danecek et al., 2011) is a tab-delimited text file format that is often used in 46 bioinformatics to store genomic variants. A VCF file consists of the header, including meta-

47 information lines and field definition lines (column names), and the body (data section). An arbitrary number of meta-information lines start with '\#\#' and provide a description of the VCF file. The body of the file consists of eight mandatory columns: chromosome (CHROM), starting position of a variant (POS), variant identifiers (ID), the reference allele (REF), a list of alternate alleles (ALT), a PHRED-scaled quality score (QUAL), filter information regarding variant validity (FILTER), and annotation information (INFO). Additional columns describing samples can also be added. Each row of the file describes specific genomic variants (SNVs, INDELs, CNVs, and other structural variants) at the given chromosome and genomic position.

VCF files often store information about numerous samples and can therefore reach huge sizes gigabytes, or sometimes terabytes. This creates an issue for the readability of VCF files and further analysis for non-programmers, as manual data extraction and analysis using Microsoft Excel or other table processing software may not be possible due to the RAM capacity limitation of standard computers. We introduce re-Searcher, bioinformatics tool specifically developed for simplified mining and analysis of big-size VCF files. We developed a multi-platform userfriendly graphical user interface (GUI) tool for offline access, while re-Searcher web application can be used online via web browser. re-Searcher has been developed for the broader biomedical community and solves the problem of working and analyzing genomics data stored in VCF format.

\section{Materials \& Methods}

\section{Implementation}

re-Searcher application was written in Python 3 (Rossum, Drake \& Van Rossum, 2010) with the implementation of Tkinter (Lundh, 1999) package to build the GUI, and Pandas (McKinney \& PyData Development Team, 2017) library to extract columns. For convenience of users who would like to build re-Searcher into their pipelines command line interface (CLI) is available as python script (see Availability). CLI mirrors functionality of GUI regarding files processing. We developed web version of re-Searcher for users to manipulate VCF files without downloading CLI or GUI versions of re-Searcher. The web application was developed using Django web framework (Django, 2013) to run python script via WSGI on Apache web server (Apache, 2020). The web version runs re-Searcher scripts on server and takes input files from website and returns processed files to user to download. re-Searcher solves the problem of analyzing large VCF files by not loading the whole file directly into RAM, but instead pre-processing it in chunks and utilizing a simple and intuitive 
80

81

82

83

84

85

86

87

88

89

90

91

92

93

94

95

96

97

98

99

100

101

102

103

104

105

106

107

108

109

110

111

112

113

114

115

116

117

118

119

interface (Figure 1). The main advantage of re-Searcher in comparison with other tools is the presence of a simple and user-friendly interface, GUI and web interface, instead of a CLI, as well as a lack of confused installation procedures typical for existing tools. The generalized workflow of re-Searcher consists of several steps: selecting an input file, setting up necessary filtering parameters, data processing, and exporting a filtered output VCF file (Figure 2). re-Searcher browses and opens VCF files with extensions ".txt" or ".vcf”, before performing the following filtering and extraction options:

\section{Header extraction}

VCF files can be large and, if a user needs to know only certain information in a file header (e.g. a particular meta-line or sample ID), the software can extract only the header from the original VCF file and save it into a new file.

\section{Keyword search}

If genomic variants need to be filtered according to the presence of a keyword, the software can find these rows and extract them into a new VCF file. Users may input multiple keywords by accessing the entry field or by uploading a .txt file with keywords.

\section{Sample extraction}

If the user needs only particular sample IDs in a VCF file, the software can extract the necessary sample columns into a new file. Similar to a keyword search, users may input multiple sample IDs by accessing the entry field or by uploading a .txt file with the IDs.

\section{Genotype format conversion}

re-Searcher can convert numeric genotype (GT) format into letter format. The conversion option is one of the most used operations when working with VCF files, for example, in further comparative analysis of genetic variants or SNPs. The original GT format in VCF files is numeric $(0 / 0,0 / 1,1 / 1$ for biallelic sites or $1 / 2,2 / 3$, etc. for multiallelic sites), where 0 is a reference (REF) allele, 1 is a first alternative (ALT) allele, 2 is a second ALT allele and so on (Danecek et al., 2011; Campbell et al., 2016). After GT format conversion REF number is replaced with REF letter and ALT number with corresponding ALT letter (Figure 3). For instance, if GT of first sample is $0 / 1$ and GT of second sample is $1 / 2$ in numeric format, while REF and two ALT are $G C, T$ and $C A A$ respectively, then after conversion first sample's letter GT becomes $G C, T$ and second sample's letter GT becomes $T$, $C A A$.

The final output file is a filtered and processed VCF file and is generated with a complement log file containing the file processing information, name of specified file and work directory with outputs.

\section{Results and Discussion}

We have compared the main features of re-Searcher with other existing and open source tools VIVA (Tollefson et al., 2019), VCFtools (Danecek et al., 2011), GEMINI (Paila et al., 2013), BrowseVCF (Salatino \& Ramraj, 2016), VCF.Filter (Müller et al., 2017), VCF-Miner (Hart et al., 2016) and prepared a detailed table (Table 1). The compared tools have been developed and 
120 implemented in different programming languages (Julia, C++, Perl, Java and Python) and

121

122

123

124

125

126

127

128

129

130

131

132

133

134

135

136

137

138

139

140

141

142

143

144

145

146

147

148

149

150

151

152

153

154

155

156

157

158

159

dedicated libraries. VCFtools is one of the most cited and advanced tools for processing VCF files, but it requires additional computational skills for effective usage. VIVA is the only tool that provides the possibility for advanced visualization and plotting figures. In addition to reSearcher, other tools with a GUI available for users are BrowseVCF, VCF.Filter, and VCFMiner, while web interface is available in only in re-Searcher (Figure 4) and GEMINI. From these, only re-Searcher, BrowseVCF and VCF.Filter tools support multiple operational systems (Windows, MacOS, Linux). Searching the whole VCF file by keyword and the corresponding extraction of data based on keywords are features available on re-Searcher and BrowseVCF, whereas genotype conversion is a unique feature of re-Searcher.

re-Searcher is a multi-platform tool and can be run on MacOS, Windows and Linux operating systems. Performance of re-Searcher has been evaluated on these PC platforms (Windows 10 Pro OS and Linux Mint 17 OS-based PC: CPU Intel Core i5-8250U 1.80 GHz, RAM 4Gb and MacOS Catalina based PC: CPU Intel Core i7, 3.2 GHz, RAM $8 \mathrm{~Gb}$ ) with different VCF file sizes. Different size VCF files $(0.081 \mathrm{~Gb}, 0.814 \mathrm{~Gb}, 1.320 \mathrm{~Gb}, 1.980 \mathrm{~Gb}$ and $7.950 \mathrm{~Gb})$ were used as input datasets for evaluating re-Searcher performance. The results of the performance benchmarking are shown in Table 2 .

We have used big VCF files from the 1000 Genomes Project (The 1000 Genomes Project Consortium, 2015) and then generated the different sized testing VCF files from this dataset. The Linux-based systems had the fastest execution time for different operations in comparison with Windows and MacOS systems.

\section{Availability}

re-Searcher executable software including source code, tested VCF files and additional information are publicly available on the GitHub repository https://github.com/LabBandSB/reSearcher. re-Searcher is free bioinformatics tool and open to all users without login and registration requirements and do not require an installation of additional tools. CLI version of reSearcher is also available on the GitHub repository for incorporation into other pipelines. In addition, web version of re-Searcher is available at https://nla-lbsb.nu.edu.kz.

\section{Conclusions}

Exploring and analyzing VCF files generated after the bioinformatics processing of sequencing data is one of the important steps performed by researchers during analysis and meta-analysis of genotype/phenotype associations. We have developed and introduced an easy-to-use bioinformatics tool, re-Searcher, with several unique features for mining big VCF files and realized with a simple graphical user interface and web interface that makes it easily available for clinicians and researchers without any computational skills. Several improvements such as visualization options (clustering and plotting functions) with Principal Component Analysis and heatmap methodologies are under future development of re-Searcher. 


\section{Acknowledgements}

161 This work is dedicated to the blessed memory of Dr.Vasily Ogryzko.

162

163

\section{References}

164

Apache HTTP Server. 2020. Apache Software Foundation.

165

166

167

168

169

170

171

172

173

174

175

176

177

178

179

180

181

182

183

Campbell IM, Gambin T, Jhangiani SN, Grove ML, Veeraraghavan N, Muzny DM, Shaw CA, Gibbs RA, Boerwinkle E, Yu F, Lupski JR. 2016. Multiallelic Positions in the Human Genome: Challenges for Genetic Analyses. Human Mutation 37:231-234. DOI: 10.1002/humu.22944.

Danecek P, Auton A, Abecasis G, Albers CA, Banks E, DePristo MA, Handsaker RE, Lunter G, Marth GT, Sherry ST, McVean G, Durbin R, 1000 Genomes Project Analysis Group. 2011. The variant call format and VCFtools. Bioinformatics 27:2156-2158. DOI: 10.1093/bioinformatics/btr330.

van Dijk EL, Jaszczyszyn Y, Naquin D, Thermes C. 2018. The Third Revolution in Sequencing Technology. Trends in Genetics 34:666-681. DOI: 10.1016/j.tig.2018.05.008.

Django. 2013. Django Software Foundation.

Gao GF, Parker JS, Reynolds SM, Silva TC, Wang L-B, Zhou W, Akbani R, Bailey M, Balu S, Berman BP, Brooks D, Chen H, Cherniack AD, Demchok JA, Ding L, Felau I, Gaheen S, Gerhard DS, Heiman DI, Hernandez KM, Hoadley KA, Jayasinghe R, Kemal A, Knijnenburg TA, Laird PW, Mensah MKA, Mungall AJ, Robertson AG, Shen H, Tarnuzzer R, Wang Z, Wyczalkowski M, Yang L, Zenklusen JC, Zhang Z, Liang H, Noble MS. 2019. Before and After: Comparison of Legacy and Harmonized TCGA Genomic Data Commons' Data. Cell Systems 9:24-34.e10. DOI:

10.1016/j.cels.2019.06.006. 
184 Goodwin S, McPherson JD, McCombie WR. 2016. Coming of age: ten years of next-generation 185 sequencing technologies. Nature Reviews Genetics 17:333-351. DOI:

186 10.1038/nrg.2016.49.

187

188

189

190

191

192

193

194

195

196

197

198

199

200

201

202

203

204

205

Hart SN, Duffy P, Quest DJ, Hossain A, Meiners MA, Kocher J-P. 2016. VCF-Miner: GUIbased application for mining variants and annotations stored in VCF files. Briefings in Bioinformatics 17:346-351. DOI: 10.1093/bib/bbv051.

Lundh F. 1999. An introduction to tkinter.

McKinney W, PyData Development Team. 2017. Pandas - Powerful Python Data Analysis Toolkit.

Molkenov A, Zhelambayeva A, Yermekov A, Mussurova S, Sarkytbayeva A, Kalykhbergenov Y, Zhumadilov Z, Kairov U. 2019. Transcriptomic Databases. In: Encyclopedia of Bioinformatics and Computational Biology. Elsevier, 341-351. DOI: 10.1016/B978-012-809633-8.20208-2.

Müller H, Jimenez-Heredia R, Krolo A, Hirschmugl T, Dmytrus J, Boztug K, Bock C. 2017. VCF.Filter: interactive prioritization of disease-linked genetic variants from sequencing data. Nucleic Acids Research 45:W567-W572. DOI: 10.1093/nar/gkx425.

Paila U, Chapman BA, Kirchner R, Quinlan AR. 2013. GEMINI: Integrative Exploration of Genetic Variation and Genome Annotations. PLoS Computational Biology 9:e1003153. DOI: 10.1371/journal.pcbi.1003153.

Rigden DJ, Fernández XM. 2020. The 27th annual Nucleic Acids Research database issue and molecular biology database collection. Nucleic Acids Research 48:D1-D8. DOI: 10.1093/nar/gkz1161. 
206 Rossum G van, Drake FL, Van Rossum G. 2010. The Python language reference. Hampton, NH:

207 Python Software Foundation.

208 Salatino S, Ramraj V. 2016. BrowseVCF: a web-based application and workflow to quickly

209 prioritize disease-causative variants in VCF files. Briefings in Bioinformatics:bbw054.

210 DOI: 10.1093/bib/bbw054.

211 The 1000 Genomes Project Consortium. 2015. A global reference for human genetic variation.

$212 \quad$ Nature 526:68-74. DOI: 10.1038/nature15393.

213 The ICGC/TCGA Pan-Cancer Analysis of Whole Genomes Consortium. 2020. Pan-cancer

214 analysis of whole genomes. Nature 578:82-93. DOI: 10.1038/s41586-020-1969-6.

215 Tollefson GA, Schuster J, Gelin F, Agudelo A, Ragavendran A, Restrepo I, Stey P, Padbury J, 216 Uzun A. 2019. VIVA (VIsualization of VAriants): A VCF File Visualization Tool.

$217 \quad$ Scientific Reports 9:12648. DOI: 10.1038/s41598-019-49114-z.

218 


\section{Figure 1}

\section{Main window of re-Searcher GUI}

\begin{tabular}{l} 
re-Searcher \\
Help \\
\hline File directory \\
\hline Keywords
\end{tabular}

Welcome to re-Searcher!

(c) Laboratory of Bioinformatics and Systems Biology Center for Life Sciences, NLA, Nazarbayev University 
Figure 2

Data processing workflow

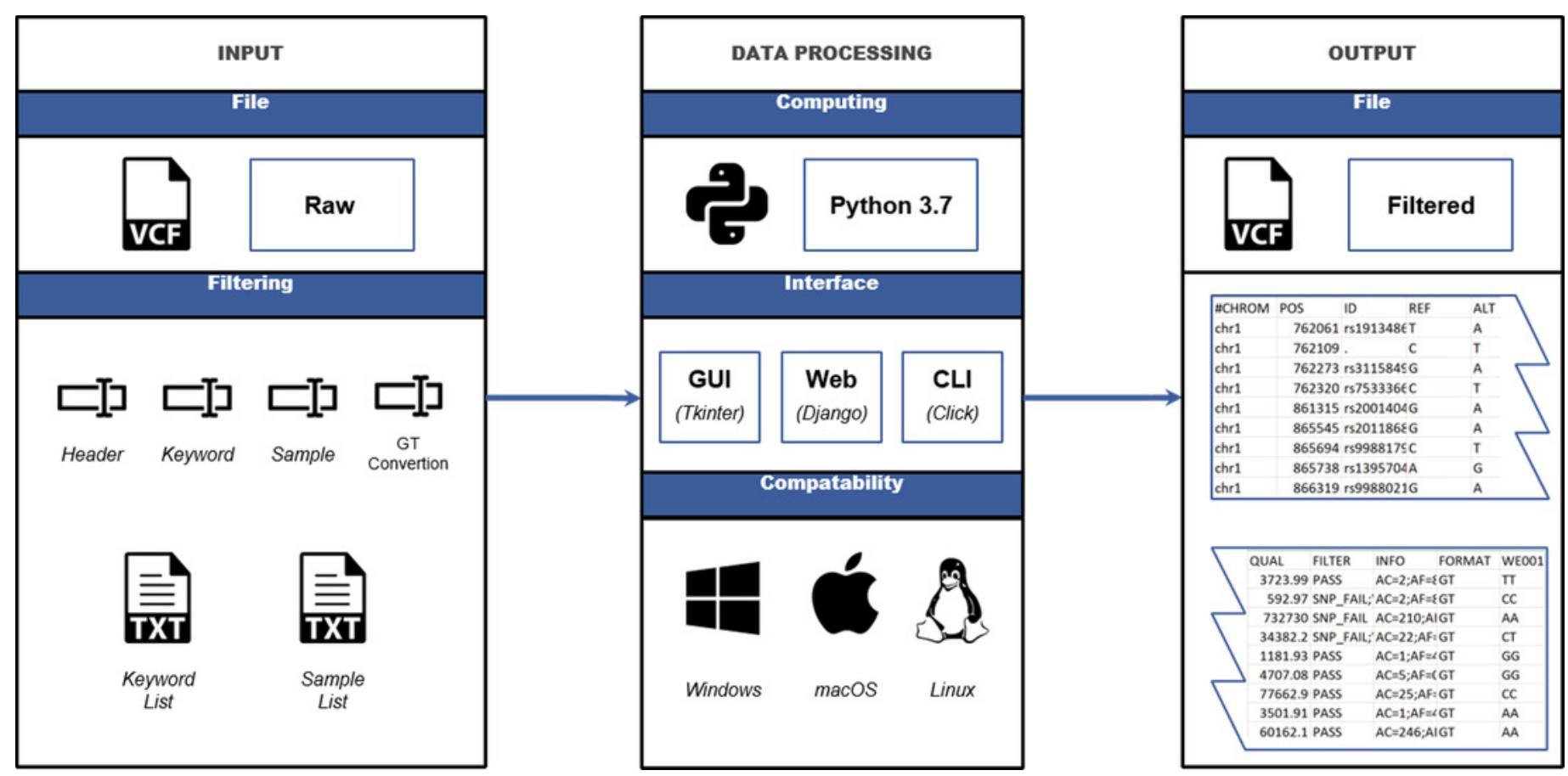




\section{Figure 3}

\section{Genotype conversion example}

\begin{tabular}{|c|c|c|c|c|c|c|c|c|c|c|c|}
\hline 4 & A & B & C & D & $E$ & $\mathrm{~F}$ & G & $\mathrm{H}$ & 1 & J & K \\
\hline 1 & \#CHROM & POS & ID & REF & ALT & QUAL & FILTER & INFO & FORMAT & HG00157 & HG00150 \\
\hline 2 & chr10 & 60494 & rs568182 & $A$ & $\mathbf{G}$ & 100 & PASS & \multicolumn{2}{|c|}{$A C=27 ; A F: G T$} & 0 & 이은 \\
\hline 3 & chr10 & 390318 & rs48811 bi & iállelic & variant & 100 & PASS & \multicolumn{2}{|c|}{$\mathrm{AC}=2549 ; \angle \mathrm{GT}$} & $1 \mid 1$ & $1 \mid 1$ \\
\hline 4 & chr10 & 614952 & rs3680122 & & A & 100 & PASS & \multicolumn{2}{|c|}{$A C=1 ; A F=(G T$} & numel & ic/GT \\
\hline 5 & chr10 & 866075 & rs2020461 & $A$ & A & 100 & PASS & \multicolumn{2}{|c|}{$A C=54 ; A F=G T$} & $0 / 0$ & $0 / 0$ \\
\hline 6 & chr1 & 151015299 & rs120670d & G & A,GA,GAA & 100 & PASS & ALC-2, 0000 & 000 & 012 & $2 / 2$ \\
\hline 7 & chr1 & 151386711 & rs53071 $\mathbf{m}$ & iültialle & lic varia & ant 100 & PASS & \multicolumn{2}{|c|}{$A C=1 ; A F=(G T$} & $0 / 0$ & $0 / 0$ \\
\hline 8 & chr1 & 151776687 & rs5572095 & & A & 100 & PASS & \multicolumn{2}{|c|}{$A C=1 ; A F=(G T$} & $0 / 0$ & $0 / 0$ \\
\hline 9 & chr1 & 152164508 & rs1121642 & & A & 100 & PASS & \multicolumn{2}{|c|}{$A C=16 ; A F ; G T$} & $0 / 0$ & $0 / 0$ \\
\hline 10 & chr1 & 152466592 & rs6668271 & & A & 100 & PASS & \multicolumn{2}{|c|}{$A C=212 ; A \mid G T$} & $0 / 0$ & $0 / 0$ \\
\hline 11 & $\operatorname{chr} 1$ & 152799940 & rs2001293 & & G & 100 & PASS & \multicolumn{2}{|c|}{$A C=303 ; A \mid G T$} & $0 / 0$ & $0 / 0$ \\
\hline 12 & $\operatorname{chr} 1$ & 153095488 & rs1824412 & & $\mathbf{T}$ & 100 & PASS & \multicolumn{2}{|c|}{$A C=1 ; A F=(G T$} & $0 / 0$ & $0 / 0$ \\
\hline \multicolumn{12}{|l|}{$\mathbf{B}$} \\
\hline 4 & A & B & C & D & & $\mathrm{F}$ & G & $\mathrm{H}$ & 1 & 1 & K \\
\hline 1 & \#CHROM & POS & ID & REF & ALT & QUAL & FILTER & INFO & FORMAT & HG00157 & HG00150 \\
\hline 2 & chr10 & 60494 & rs568182d & A & G & 100 & PASS & \multicolumn{2}{|c|}{$A C=27 ; A F=G T$} & A/A & $A / A$ \\
\hline 3 & chr10 & 390318 & rs4881153 & A & G & 100 & PASS & \multicolumn{2}{|c|}{$A C=2549 ; \angle G T$} & $\mathrm{G} / \mathrm{G}$ & $\mathrm{G} / \mathrm{G}$ \\
\hline 4 & chr10 & 614952 & rs3680122 & G & A & 100 & PASS & \multicolumn{2}{|c|}{$A C=1 ; A F=(G T$} & let & scG \\
\hline 5 & chr10 & 866075 & rs2020461 & $A$ & A & 100 & PASS & \multicolumn{2}{|c|}{$A C=54 ; A F=G T$} & $\mathrm{~A} / \mathrm{A}$ & $A / A$ \\
\hline 6 & chr1 & 151015299 & rs 1206706 & $G$ & $A, G A, G A A$ & 100 & PASS & $A C=2,550 C$ & 001 & G/GA & $\mathrm{GA} / \mathrm{GA}$ \\
\hline 7 & chr1 & 151386711 & rs5307160 & & G & 100 & PASS & \multicolumn{2}{|c|}{$A C=1 ; A F=(G T$} & $T / T$ & $\mathrm{~T} / \mathrm{T}$ \\
\hline 8 & chr1 & 151776687 & rs5572095 & G & A & 100 & PASS & \multicolumn{2}{|c|}{$A C=1 ; A F=(G T$} & G/G & G/G \\
\hline 9 & chr1 & 152164508 & rs1121642 & G & A & 100 & PASS & \multicolumn{2}{|c|}{$A C=16 ; A F ; G T$} & G/G & G/G \\
\hline 10 & chr1 & 152466592 & rs6668271 & G & A & 100 & PASS & \multicolumn{2}{|c|}{$A C=212 ; A \mid G T$} & G/G & G/G \\
\hline 11 & chr1 & 152799940 & rs2001293 & A & G & 100 & PASS & \multicolumn{2}{|c|}{$A C=303 ; A \mid G T$} & $A / A$ & $A / A$ \\
\hline 12 & chr1 & 153095488 & rs1824412 & & $\mathrm{T}$ & 100 & PASS & \multicolumn{2}{|c|}{$A C=1 ; A F=(G T$} & $\mathrm{c} / \mathrm{c}$ & $\mathrm{c} / \mathrm{C}$ \\
\hline
\end{tabular}




\section{Figure 4}

Web interface of re-Searcher available via browser at https://nla-lbsb.nu.edu.kz

\section{re-Searcher}

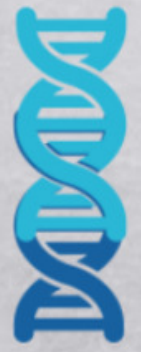

re-Searcher is a toolbox aimed to simplify the task for genomics data mining from VCF files. Now there's no need to perform difficult script manipulations in IDE with Python or R. re-Searcher can work with any variant of VCF, for instance, with annotated VCF in ANNOVAR.

Source code available on GitHub

\section{Filter VCF files}

Samples:
Keywords: $\quad$ Keyword1 Keyword2 Keyword3
Chromosomes:

$$
\text { Positions: } 10002000
$$

Extract header without meta-information lines

Choose File No file chosen

Filter

\section{Contacts}

re-Searcher was created in Laboratory of Bioinformatics and Systems Biology, Center for Life Sciences, National Laboratory AstanaNazarbayev University
Extract Headers From VCF file

Extract header without metainformation lines

Choose File No file chosen

Extract Headers
Convert The Numeric GT Format To Letter GT Format

Extract header without metainformation lines

Choose File No file chosen 


\section{Table 1 (on next page)}

Comparison of re-Searcher's features with similar tools 
1 Table 1. Comparison of re-Searcher's features with similar tools

2

\begin{tabular}{|c|c|c|c|c|c|c|c|c|}
\hline Categories & Features & $\begin{array}{c}\text { re- } \\
\text { Searcher }\end{array}$ & VIVA & VCFtools & GEMINI & BrowseVCF & VCF.Filter & $\begin{array}{l}\text { VCF- } \\
\text { Miner }\end{array}$ \\
\hline \multirow{5}{*}{$\begin{array}{c}\text { Technical } \\
\text { Aspects }\end{array}$} & $\begin{array}{c}\text { Compatibility } \\
\text { with } \\
\text { operation } \\
\text { system } \\
\end{array}$ & $\begin{array}{l}\text { Windows, } \\
\text { MacOS, } \\
\text { Linux }\end{array}$ & $\begin{array}{c}\text { Windows, } \\
\text { MacOS, } \\
\text { Linux }\end{array}$ & $\begin{array}{c}\text { Windows, } \\
\text { MacOS, } \\
\text { Linux }\end{array}$ & $\begin{array}{c}\text { Windows, } \\
\text { MacOS, } \\
\text { Linux }\end{array}$ & $\begin{array}{l}\text { Windows, } \\
\text { MacOS, } \\
\text { Linux }\end{array}$ & $\begin{array}{l}\text { Windows, } \\
\text { MacOS, } \\
\text { Linux }\end{array}$ & Windows \\
\hline & Language & Python & Julia & $\mathrm{C}++$, Perl & Python & $\begin{array}{c}\text { Python, } \\
\text { JavaScript, } \\
\text { CSS, } \\
\text { HTML5 }\end{array}$ & Java & Java \\
\hline & Interface & $\begin{array}{c}\text { GUI, } \\
\text { Web } \\
\text { Browser, } \\
\text { CLI }\end{array}$ & $\begin{array}{c}\text { CLI, } \\
\text { Jupyter } \\
\text { Notebook }\end{array}$ & CLI & $\begin{array}{l}\text { Web } \\
\text { Browser, } \\
\text { CLI }\end{array}$ & $\begin{array}{l}\text { GUI, } \\
\text { CLI }\end{array}$ & GUI & GUI \\
\hline & $\begin{array}{l}\text { Works } \\
\text { offline }\end{array}$ & $V$ & $V$ & $V$ & $X$ & $X$ & $V$ & $V$ \\
\hline & $\begin{array}{l}\text { Portable } \\
\text { launcher }\end{array}$ & $V$ & $X$ & $X$ & $X$ & $V$ & $X$ & $X$ \\
\hline \multirow{5}{*}{ Functionality } & $\begin{array}{l}\text { Search by } \\
\text { keyword }\end{array}$ & $V$ & $X$ & $X$ & $X$ & $V$ & $X$ & $X$ \\
\hline & $\begin{array}{c}\text { Sample } \\
\text { selection }\end{array}$ & $V$ & $V$ & $V$ & $V$ & $V$ & $V$ & $V$ \\
\hline & $\begin{array}{c}\text { Genotype } \\
\text { format } \\
\text { conversion }\end{array}$ & $V$ & $X$ & $X$ & $X$ & $X$ & $X$ & $X$ \\
\hline & Visualization & $X$ & $V$ & $X$ & $X$ & $X$ & $X$ & $X$ \\
\hline & $\begin{array}{c}\text { Export } \\
\text { filtered VCF } \\
\text { file }\end{array}$ & $V$ & $X$ & $V$ & $X$ & $X$ & $V$ & $V$ \\
\hline
\end{tabular}


Table 2 (on next page)

re-Searcher multi-platform run time comparison 
1 Table 2. re-Searcher multi-platform run time comparison

2

3 OS - operational system, GT - genotype, sec - seconds, Gb - gigabyte.

4

\begin{tabular}{|c|c|c|c|c|c|}
\hline \multirow[b]{2}{*}{$\mathrm{OS}$} & \multirow{2}{*}{$\begin{array}{c}\text { VCF file size } \\
\text { (Gb) }\end{array}$} & \multicolumn{4}{|c|}{ Execution Time (sec) } \\
\hline & & Extract header & $\begin{array}{l}\text { Keyword } \\
\text { extraction }\end{array}$ & $\begin{array}{l}\text { Sample ID } \\
\text { extraction }\end{array}$ & $\begin{array}{c}\text { GT } \\
\text { conversion }\end{array}$ \\
\hline \multirow{5}{*}{ Linux } & 0.081 & 2.227 & 9.995 & 15.243 & 38.375 \\
\hline & 0.814 & 2.497 & 38.106 & 21.334 & 117.186 \\
\hline & 1.320 & 2.462 & 67.260 & 61.159 & 206.868 \\
\hline & 1.980 & 2.115 & 75.331 & 104.167 & 330.527 \\
\hline & 7.950 & 6.145 & 366.137 & 200.347 & 1117.168 \\
\hline \multirow{5}{*}{ Windows } & 0.081 & 14.865 & 22.482 & 4.785 & 49.642 \\
\hline & 0.814 & 18.898 & 48.820 & 21.398 & 139.641 \\
\hline & 1.320 & 21.054 & 44.669 & 59.329 & 238.192 \\
\hline & 1.980 & 10.919 & 53.958 & 90.446 & 339.275 \\
\hline & 7.950 & 16.308 & 502.996 & 309.177 & 1320.197 \\
\hline \multirow{5}{*}{ MacOS } & 0.081 & 9.627 & 9.297 & 10.423 & 16.298 \\
\hline & 0.814 & 5.262 & 20.916 & 19.705 & 116.82 \\
\hline & 1.320 & 6.286 & 35.433 & 53.247 & 186.181 \\
\hline & 1.980 & 3.231 & 37.923 & 119.136 & 286.544 \\
\hline & 7.950 & 5.457 & 148.612 & 254.128 & 1130.225 \\
\hline
\end{tabular}

5

6

7

8 